\title{
Preparation and Characteristics of Film Dosage from Natural Polysaccharides
}

\author{
Yoshifumi Murata $^{{ }^{*}}$, Kyoko Kofuji ${ }^{1}$, Norihisa Nishida ${ }^{2}$, Ryosei Kamaguchi ${ }^{2}$ \\ ${ }^{1}$ Faculty of Pharmaceutical Science, Hokuriku University, Kanazawa, Japan; ${ }^{2}$ Morishita Jintan Co. Osaka Technocenter, Hirakata, \\ Japan. \\ Email: y-murata@hokuriku-u.ac.jp
}

Received May $2^{\text {nd }}$, 2011; revised June $2^{\text {nd }}, 2011$; accepted June $10^{\text {th }}, 2011$.

\begin{abstract}
We investigated preparation of film dosage form (FD) from natural polysaccharides using the casting method without organic solvents, heating or $\mathrm{pH}$ control. Ferulic acid (FA) and catechin were employed as model compounds incorporated in the FD, and the release profile of each compound from the form was investigated in the limited medium. Film formation was affected by the addition of the model compound to the polysaccharide solution. Rigid FD was obtained with 2\% low-molecular-weight alginate ( $L-A L G$; thickness, $65 \mu \mathrm{m}$ ), and it hardened after the addition of 0.5\% polygalacturonic acid, although the thickness of the film did not change. The FDs immediately released the model compound, and the forms dissolved in phosphate-buffered saline. FD modification did not affect the FA release rate except in the early stage. FD would be a useful dosage form, especially for preventing or treating localized problems in the oral cavity.
\end{abstract}

Keywords: Film Dosage Form, Natural Polysaccharide, Sodium Alginate, Ferulic Acid, Catechin

\section{Introduction}

Film has been noted as a dosage form in the fields of medicine and cosmetics [1,2]. The film dosage form (FD) adheres to a surface when a little liquid is present on it, and then the drug or another active compound incorporated in the film is released. The FD design results in the distribution of the drug across the region to which the form is attached. However, FD swells and erodes if it is prepared with aqueous-soluble materials. For example, fast-dissolving film is quickly disintegrated by saliva when it is used in the oral cavity [3,4]. Recently, this film has attracted interest not only for oral care but for patients with aphagia or dysphagia as well $[5,6]$.

Polymer compounds are generally utilized as the film's base. Some water-soluble polymers are especially useful because of the safety in which the film is prepared and applied to humans. For example, gelatin polypeptide is the most popular one for film formation and has been used as the base for capsule preparation [7]. First, aqueous gelatin solution is prepared, and then, a cross- linking agent such as glutaraldehyde is added to stabilize the film. Agar, a polysaccharide has also been studied as a potential material for film preparation. In general, agar dissolves in water by heating, and the resultant solution is then cooled and dried for film formation. In the case of
FD preparation, active compounds such as drugs are added to the polymer solution. Therefore, the chemical or thermal stability of the compound must be incorporated when FD is prepared using these polymers. On the other hand, some polysaccharides can dissolve in aqueous solution without heat and form the film in dry solvent. For example, neutral polysaccharides such as pullulan (PUL) and acidic polysaccharides such as sodium alginate (ALG) and sodium chondroitin sulfate (CHS) are known film bases [8-11]. Although FD is readily prepared using these aqueous polysaccharides, film formation is affected in the presence of additives, including drugs.

In this study, we investigated the preparation of FD from natural polysaccharides without dissolving in organic solvents, heating, controlling the $\mathrm{pH}$ or adding a plasticizer such as sorbitol, fatty acid or polyvinyl alcohol. Ferulic acid (FA) and catechin (CA) were employed as model compounds. FA is an anti-oxidizing agent and a part of the human diet [12], and its physiological action mitigates lifestyle-related diseases such as hypertension, hyperlipidemia, and diabetes [13-15]. CA is a polyphenol compound and a component of green tea that used for beverages [16]. The model compound is expected to be active following dissolution in body fluids such as saliva 
upon oral FD administration [17,18]. Therefore, the release profile of each FD compound was investigated in limited dissolution medium.

\section{Experimental}

\subsection{Materials}

Low-molecular-weight alginate (L-ALG) was obtained from Alfa Aesar (Ward Hill, MA, USA), and high-molecular weight alginate (H-ALG), from Nacalai Tesque Inc. (300 cps, Kyoto, Japan). Pullulan (PUL) was supplied by Hayashibara Biochemical Laboratories (Okayama, Japan), and a polysaccharide produced by Bifidobacterium longum JBL05 (BPS) was supplied by Morishita Jintan (Osaka, Japan) [19]. CHS (C type) and phos- phate-buffered saline (PBS; pH 7.4) were purchased from Wako Pure Chemicals (Osaka, Japan). Chitosan (CS; degree of deacetylation: 75\% - 85\%) was obtained from Kimitsu Chem. Ind. (Tokyo, Japan). Hydroxypropyl methylcellulose acetate succinate (AS-HF type; HPMC) was obtained from Shinetsu Chem. Co. (Tokyo, Japan). A polygalacturonic acid (PGA) was purchased from MP Biomedicals (Solon, OH, USA) and another (pectic acid) from Wako Pure Chemical. FA was purchased from Tsuno Food Ind. Co. Ltd. (Wakayama, Japan). (+)-Catechin hydrate (CA) was purchased from Spectrum Chemical MFG. Co. (New Brunswick, NJ, USA). All other chemicals were of reagent grade.

\subsection{FD Preparation}

Each polysaccharide solution $(0.5 \%$ - $4 \%)$ was prepared using deionized water. A model compound was added to the polysaccharide solution and mixed well, and $3.0 \mathrm{~g}$ of the solution was poured into a plastic Petri dish (diameter, $54 \mathrm{~mm}$ ). After $24 \mathrm{~h}$ at $37^{\circ} \mathrm{C}$, the film formed on the dish was transferred into a desiccator. Film formation was judged to not have occurred if a film could not be removed from the bottom of the dish.

\subsection{Film Thickness and Rheological Properties}

Film thickness was measured at 10 points on each film using a micrometer (CLM1-15QM; Mitutoyo, Kawasaki, Japan) with a set pressure of $0.5 \mathrm{~N}$. Measurements were made on 3 films, and the mean thickness was calculated. The rheological properties of each film were determined using a rheometer (SUN RHEO TEX SD-700\#; Sun Scientific Co., Tokyo, Japan) at room temperature. The film was fixed on a vial (inner diameter, $1.4 \mathrm{~mm}$; outer diameter, $18.8 \mathrm{~mm}$ ) using joining tape (Scotch mending tape; Sumitomo 3M Ltd., Tokyo, Japan) and probed with a cylindrical adapter (diameter, $5.0 \mathrm{~mm}$ ). Stress and strain were measured at the point at which the adapter broke through the film. All tests were performed in trip- licate.

\subsection{X-Ray Diffractometry}

X-ray diffractometry was carried out using an automatic diffractometer (D8 DISCOVER with GADDS; Bruker AXS K.K., Yokohama, Japan) with a voltage of $40 \mathrm{kV}$ and a current of $40 \mathrm{~mA}$. The results of X-ray diffraction were interpreted using a computer program (Bruker AXS K.K.).

\subsection{Dissolution Test}

Physiological saline or PBS (pH 7.4) was used as the dissolution test medium. A film was placed in a plastic dish, and $10 \mathrm{~mL}$ of dissolution medium incubated at $37^{\circ} \mathrm{C}$ was added. The dish was shaken at $300 \mathrm{rpm}$ in a shaker incubator at $37^{\circ} \mathrm{C}$. An $80 \mu \mathrm{L}$ aliquot was removed periodically and placed in a micro test tube $(1.5 \mathrm{~mL})$ and $720 \mu \mathrm{L}$ of methanol was added to precipitate the polysaccharide dissolved from the dosage form. The sample was mixed and centrifuged (10,000 rpm, $5 \mathrm{~min}$ ), and then, the supernatant was injected into a high-performance liquid chromatography (HPLC) column. All tests were performed in triplicate. The HPLC system had an LC-6A pump (Shimadzu Co., Kyoto), a packed column $(150 \mathrm{~mm} \times 4.6 \mathrm{~mm}$; Cosmosil 5C 18 -MS-II; Nacalai Tesque, Kyoto), and a SPD-6A UV detector (Shimadzu Co.). For determination of FA, HPLC was conducted at an ambient temperature using an eluent containing 10 $\mathrm{mmol} / \mathrm{L}$ phosphate buffer ( $\mathrm{pH} 4.7$ ) and methanol (8:2) at a flow rate of $0.8 \mathrm{~mL} / \mathrm{min}$ [20]. The detector wavelength was set as $260 \mathrm{~nm}$, and an eluent comprising $0.1 \%$ citric acid and acetonitrile (87:13) was used to quantify CA; the detector wavelength was then set to $280 \mathrm{~nm}$ [21].

\section{Results and Discussions}

To form circular film using the casting method, 1 - 4\% solution of each polysaccharide was prepared without heating and poured into a Petri dish. The solvent was then evaporated. The film formation was affected by adding FA or CA to the polymer solution. Neither 4\% CHS nor $1.5 \%$ H-ALG adequately utilized the FD materials containing $3 \mathrm{mg}$ FA. In addition, 4\% CHS did not form a circular film, while $1.5 \% \mathrm{H}-\mathrm{ALG}$ formed a fragile film (Figure 1). On the other hand, 4\% PUL formed a rigid film for which the thickness and the stress were 51 $\pm 2 \mu \mathrm{m}$ and $>500 \mathrm{kPa}$, respectively. Additionally, 0.5\% BPS formed a soft film (thickness, $52 \pm 2 \mu \mathrm{m}$; stress, $50 \mathrm{kPa}$ ). In the case of $2 \% \mathrm{~L}-\mathrm{ALG}$, a circular film (thickness, $65 \pm 1 \mu \mathrm{m}$ ) was obtained that could be modified through addition of other polysaccharides such as PGA. Both 4\% PUL and 2\% L-ALG formed circular films when CA was include in the film base (Figure 2). 

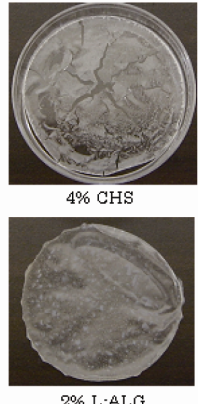

$2 \%$ L-ALG

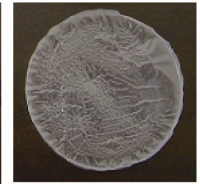

$1.5 \% \mathrm{H} \cdot \mathrm{ALG}$

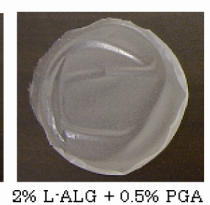

$2 \% \mathrm{~L} \cdot \mathrm{ALG}+0.5 \% \mathrm{PGA}$

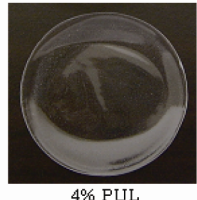

$4 \%$ PUL

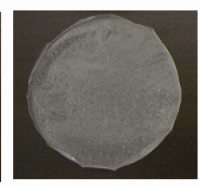

$2 \% \mathrm{~L} \cdot \mathrm{ALG}+1 \% \mathrm{CS}$
Figure 1. FDs prepared using various polysaccharides containing FA (3 mg).

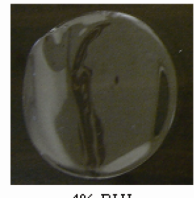

$4 \%$ PUL

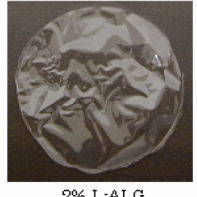

$2 \% \mathrm{~L} \cdot \mathrm{ALG}$

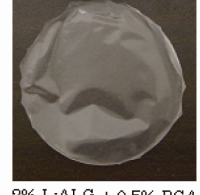

$2 \% \mathrm{~L} \cdot \mathrm{ALG}+0.5 \% \mathrm{PGA}$
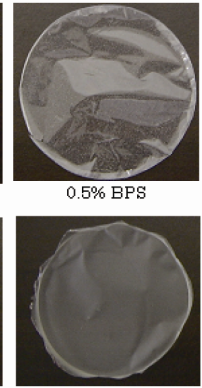

Figure 2. FDs prepared using various polysaccharides containing CA (1.5 mg).

Table 1. FD thicknesses prepared using various polysaccharides containing 3 mg FA.

\begin{tabular}{cccc}
\hline Film base & Additive & Thickness $(\mu \mathrm{m})$ & SD $(\mu \mathrm{m})$ \\
\hline 2\% L-ALG & & 65 & 1 \\
& $0.5 \%$ CS & 96 & 6 \\
& $1 \%$ CS & 144 & 3 \\
& 1\% EC & 87 & 2 \\
& 1\% HPMC & 56 & 5 \\
& 1\% CHS & 55 & 4 \\
& 1\% pectic acid & 60 & 2 \\
& 0.1\% PGA & 70 & 1 \\
& 0.5\% PGA & 67 & 4 \\
& 1\% PGA & 71 & 3 \\
& 1.5\% PGA & 75 & 1 \\
3\% L-ALG & 2\% PGA & 70 & 0 \\
\hline $.5 \%$ H-ALG & 1\% PGA & 49 & 8 \\
\hline
\end{tabular}

The actual drug contents calculated by the weights of dried films were $6.6 \%$ (1.5\% H-ALG), 4.2\% (2\% L-ALG) and $2.5 \%$ (4\% PUL), respectively.

Table 1 shows the thickness of the film prepared using L-ALG or H-ALG as a film base. The thicknesses of the films prepared with $2 \%$ L-ALG containing each polysaccharide except for $1 \%$ CS were $<100 \mu \mathrm{m}$. When FD was prepared using $2 \%$ L-ALG thickened by the addition of $0.5 \%$ CS; however, the increment of thickness did not necessarily increase FD hardness (Figure 3). A more rigid film was obtained by the addition of $0.5 \%$ PGA to

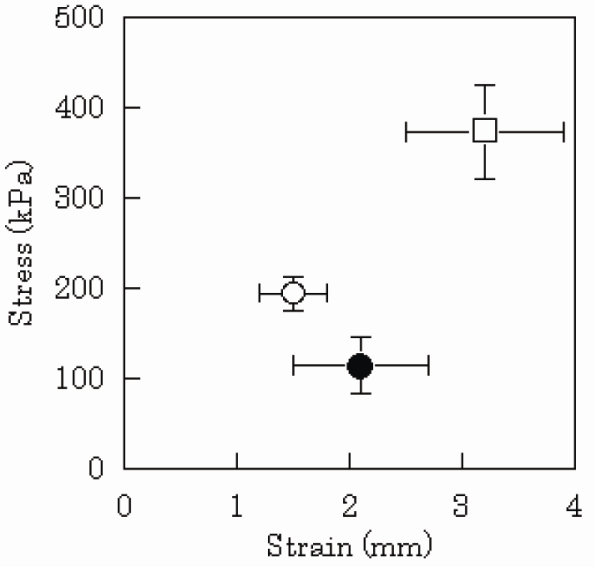

Figure 3. Rheological properties of FDs containing $3 \mathrm{mg}$ FA. Closed circle: $2 \%$ L-ALG; open circle: $2 \%$ L-ALG + $0.5 \%$ CS; open square: $2 \%$ L-ALG + $0.5 \%$ PGA.

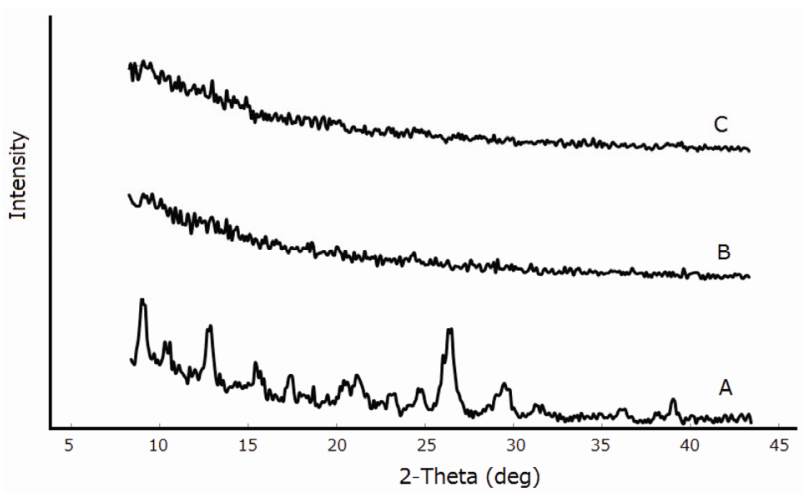

Figure 4. X-ray diffractograms. A: FA (powder); B: 1.5\% H-ALG film (FA free); C: $1.5 \%$ H-ALG film containing FA.

2\% L-ALG, although film thickness did not change.

Figure 4 shows the $\mathrm{X}$-ray diffraction patterns obtained from FA powder and FDs prepared with 1.5\% H-ALG; FA exhibited a characteristic crystalline compound pattern of diffraction. On the other hand, FD containing FA showed a pattern which lacked the characteristic diffraction peaks of FA. This result shows that the crystal form of FA is only slightly present in FD.

FD is expected to release the compound contained in the dosage form upon contact with saliva, which is secreted from the salivary glands at 0.5 - $0.6 \mathrm{~L} /$ day $(1.5$ $2.0 \mathrm{~mL} / \mathrm{min}$ when stimulated) [22]. In this study, a film was soaked in $10 \mathrm{~mL}$ of PBS buffer (pH 7.4), and the released amount of FA or CA was then measured. The release profiles of FA from the FDs prepared using the different polysaccharides are shown in Figure 5. All FDs subsequently swelled in the dissolution medium and released FA incorporated in the FDs with disintegration. In particular, FD prepared with H-ALG or BPS quickly 


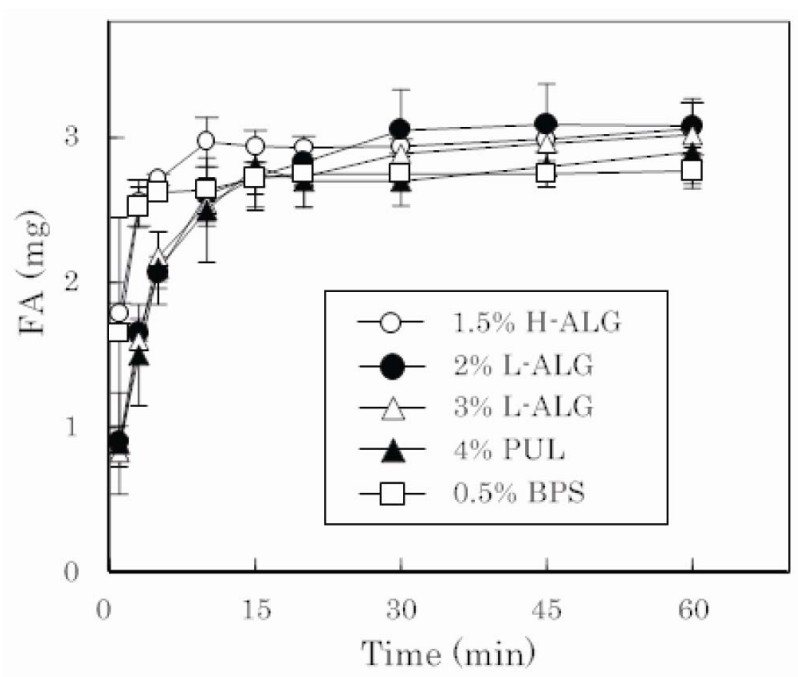

Figure 5. Release profiles of FA from FDs in PBS (pH 7.4).
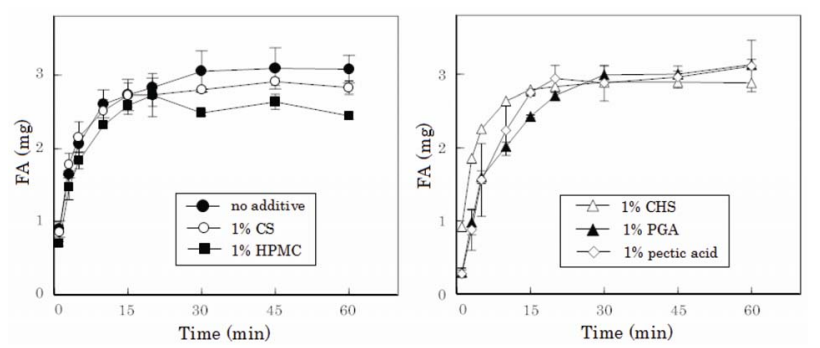

Figure 6. The release profile of FA from FDs prepared with 2\% L-ALG.

dissolved and released the total amount of FA at 5 - 10 min. When $2 \%$ L-ALG or $4 \%$ PUL was used, the FA was released for about $30 \mathrm{~min}$. A similar release profile was obtained using FD prepared with 3\% L-ALG. In FDs, model compounds such as FA are dispersed in watersoluble polymer matrices, and the FA particles may dissolve in the dissolution medium as the film erodes. The release rate was affected by the property of the compound incorporated in the FD. CA was also released immediately; for example, the total amount contained in the FD prepared with $2 \%$ L-ALG was released at $10 \mathrm{~min}$ (data not shown). The release profile was attributed to the high solubility of CA in the dissolution medium, and the rapid release rate was also observed from the FD prepared with $4 \%$ PUL.

FD prepared with $2 \%$ L-ALG that was modified through addition of CS, HPMC, or CHS did not affect FA release (Figure 6). On the other hand, the initial release rate of FA was depressed when FD modified through the addition of PGA. A similar additive effect was observed in FD modified with pectic acid. Depression of the FA release rate through the addition of PGA to $2 \%$ L-ALG was restricted at an extremely early stage,

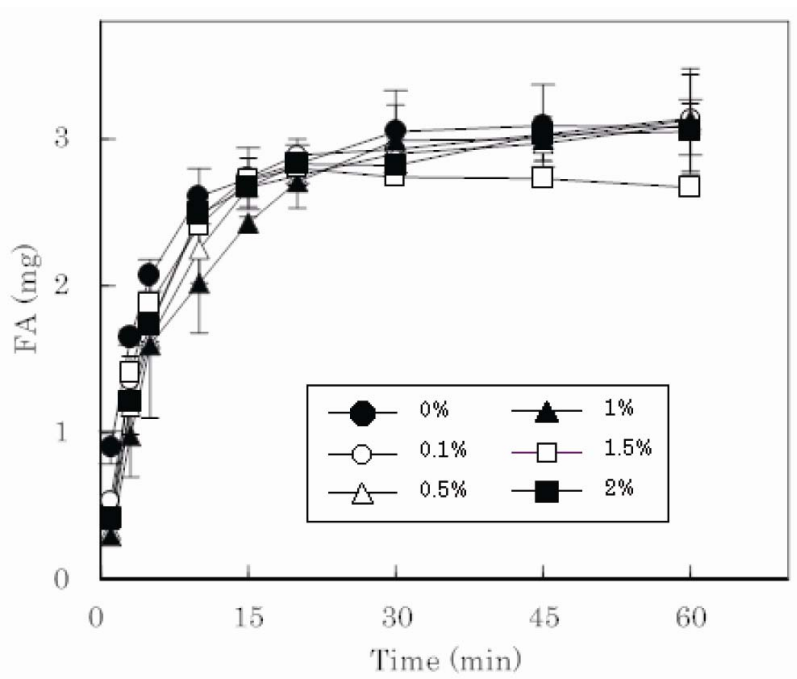

Figure 7. Effect of PGA concentration on the release profile of FA from FDs prepared with 2\% L-ALG.

and the rapid release was observed after the film swelled (Figure 7).

\section{Conclusions}

In this study, FDs were prepared using natural polysaccharides without dissolving them into organic solvents, heating them, controlling their $\mathrm{pH}$, or adding plasticizers. Some of these polysaccharides were able to incorporate model compounds such as FA or CS, and all FDs immediately released them as the forms eroded in the limited dissolution medium. FD is a useful dosage form, especially for preventing or treating localized problems in the oral cavity including dental caries and periodontal diseases. However, the drug loading capacity of FD is typically very low; therefore, the compounds incorporated in the FD should be carefully selected.

\section{Acknowledgements}

The authors would like to thank Dr. M Kimizu (Industrial Research Institute of Ishikawa) for his help and advice on X-ray Diffractometry experiment.

\section{References}

[1] E. M. Hoffmann, A. Breitenbach and J. Breitkreutz, "Advances in Orodispersible Films for Drug Delivery," Expert Opinion on Drug Delivery, Vol. 8, No. 3, 2011, pp. 299-316. doi:10.1517/17425247.2011.553217

[2] A. Nishida, M. Yamada, T. Kanazawa, Y. Takashima, K. Ouchi and H. Okada, "Sustained-Release of Protein from Biodegradable Sericin Film, Gel and Sponge,” International Journal of Pharmaceutics, Vol. 407, 2011, pp. 4452. doi:10.1016/j.ijpharm.2011.01.006

[3] S. Kunte and P. Tandale, "Fast Dissolving Strips: A Novel Approach for the Drug Delivery of Verapamil,” Jour- 
nal of Pharmacy and Bioallied Sciences, Vol. 2, No. 4, 2010, pp. 325-328. doi:10.4103/0975-7406.72133

[4] Y. Murata, T. Isobe, K. Kofuji, N. Nishida and R. Kamaguchi, "Preparation of Fast Dissolving Films for Oral Dosage from Natural Polysaccharides,” Materials, Vol. 3, 2010, pp. 4291-4299. doi:10.3390/ma3084291

[5] M. Nishimura, K. Matsuura, T. Tsukioka, H. Yamashita, N. Inagaki, T. Sugiyama and Y. Itoh, "In vitro and in Vivo Characteristics of Prochlorperazine Oral Disintegration Film,” International Journal of Pharmaceutics, Vol. 368, 2009, pp. 98-102. doi:10.1016/j.ijpharm.2008.10.002

[6] Y. Sakuda, A. Ito, M. Sasatsu and Y. Machida, "Preparation and Evaluation of Medical Carbon Oral Films," Chemical and Pharmaceutical Bulletin, Vol. 58, No. 4, 2010, pp. 454-457. doi:10.1248/cpb.58.454

[7] M. F. Senyucel, M. B. Kologlu, R. Vargun, C. Akbay, F. N. Sarac, N. Renda, N. Hasirci, G. Gollu and H. Dindar, "The Effect of Local and Sustained Release of Fibroblast Growth Factor on Wound Healing in Esophageal Anastomoses," Journal of Pediatric Surgery, Vol. 43, 2008, pp. 290-295. doi:10.1016/j.jpedsurg.2007.10.016

[8] K. C. Wood, J. Q. Boedicker D. M. Lynn and P. T. Hammond, "Tunable Drug Release from Hydrolytically Degradable Layer-By-Layer Thin Film,” Langmuir, Vol. 21, No. 4, 2005, pp. 1603-1609. doi:10.1021/la0476480

[9] P. Sriamornsak and R. A. Kennedy, "Swelling and Diffusion Studies of Calcium Polysaccharide Gels Intended for Film Coating," International Journal of Pharmaceutics, Vol. 358, 2008, pp. 205-213. doi:10.1016/j.ijpharm.2008.03.009

[10] Y. Sakata and M. Otsuka, "Evaluation of Relationship between Molecular Behavior and Mechanical Strength of Pullulan Films," International Journal of Pharmaceutics, Vol. 374, 2009, pp. 33-38. doi:10.1016/j.ijpharm.2009.02.019

[11] A. M. Smith, A. Ingham, L. M. Grover and Y. Perrie, "Polymer Film Formulations for the Preparation of Enteric Pharmaceutical Capsules,” Journal of Pharmacy and Pharmacology, Vol. 62, 2010, pp. 167-172. doi:10.1211/jpp.62.02.0003

[12] J. Alamed, W. Chaiyasit, D. J. McClements and E. A. Decker, "Relationships between Free Radical Scavenging and Antioxidant Activity in Foods," Journal of Agricultural and Food Chemistry, Vol. 57, No. 7, 2009, pp. 2969-2976. doi:10.1021/jf803436c

[13] M. Ohnishi, T. Matsuo, T. Tsuno, A. Hosoda, E. Nomura,
H. Taniguchi, H. Sasaki and H. Morishita, “Antioxidant Activity and Hypoglycemic Effect of Ferulic Acid in STZ-Induced Diabetic Mice and KK-Ay Mice,” Biofactors, Vol. 21, No. 1-47, 2004, pp. 315-319.

[14] A. Suzuki, D. Kagawa, A. Fujii, R. Ochiai, I. Tokimitsu and I. Saito, "Short- and Long-Term Effects of Ferulic Acid on Blood Pressure in Spontaneously Hypertensive Rats," American Journal of Hypertension, Vol. 15, No. 4, 2002, pp. 351-357. doi:10.1016/S0895-7061(01)02337-8

[15] B. Wang, J. Ouyang, Y. Liu, J. Yang, L. Wei, K. Li and H. Yang, "Sodium Ferulate Inhibits Atherosclerogenesis in Hyperlipidemia Rabbits," Journal of Cardiovascular Pharmacology, Vol. 43, No. 4, 2004, pp. 549-554. doi:10.1097/00005344-200404000-00010

[16] K. Hamaishi, R. Kojima and M. Ito, “Anti-Ulcer Effect of Tea Catechin In Rats,” Biological and Pharmaceutical Bulletin, Vol. 29, No. 11, 2006, pp. 2206-2213. doi:10.1248/bpb.29.2206

[17] X. Xu, X. D. Zhou and C. D. Wu, "The Tea Catechin Epigallocatechin Gallate Suppresses Cariogenic Virulence Factors of Streptococcus Mutans," Antimicrobial Agents and Chemotherapy, Vol. 55, No. 3, 2011, pp. 1229-1236. doi:10.1128/AAC.01016-10

[18] S. M. S. Miguel, L. A. Opperman, E. P. Allen, J. Zielinski and K. K. H. Svoboda, "Antioxidants Counteract Nicotine and Promote Migration via RacGTP in Oral Fibroblast Cells,” Journal of Periodontology, Vol. 81, No. 11, 2010, pp. 1675-1690. doi:10.1902/jop.2010.100187

[19] M. Kohno, S. Suzuki, T. Kanaya, T. Yoshino, Y. Matsuura, M. Asada and S. Kitamura, "Structural Characterization of the Extracellular Polysaccharide Produced by Bifidobacterium Longum JBL05," Carbohydate Polymers, Vol. 77, 2009, pp. 351-357. doi:10.1016/j.carbpol.2009.01.013

[20] Y. Murata, K. Nagaki, K. Kofuji and T. Kishi, "Functions of Chitosan-Ferulic Acid Salt for Prevention of Hypertension," Food Science and Technology Research, Vol. 16, No. 5, 2010, pp. 437-442. doi:10.3136/fstr.16.437

[21] T. Fu, J. Liang, G. Han, L. Lv and N. Li, "Simultaneous Determination of the Major Active Components of Tea Polyphenols in Rat Plasma by a Simple and Specific HPLC Assay,” Journal of Chromatography B, Vol. 875, 2008, pp. 363-367. doi:10.1016/j.jchromb.2008.09.027

[22] B. Nauntofte, J. O. Tenovuo and F. Lagerlof, "Secretion and Composition of Saliva," In: O. Fejerskov and E. A. M. Kidd, Eds., Dental Caries the Disease and Its Clinical Management, Chapter 2, Blackwell Munksgaad, Oxford, 2003, pp. 7-27. 